\title{
Nursing home-acquired pneumonia presenting at the emergency department
}

\author{
Rui Pereira $^{1}$ (I) $\cdot$ Sara Oliveira ${ }^{2} \cdot$ André Almeida $^{2}$
}

Received: 11 September 2015/ Accepted: 13 February 2016

(C) SIMI 2016

\begin{abstract}
Nursing home-acquired pneumonia (NHAP) is one of the most common infections arising amongst nursing home residents, and its incidence is expected to increase as population ages. The NHAP recommendation for empiric broad-spectrum antibiotic therapy, arising from the concept of healthcare-associated pneumonia, has been challenged by recent studies reporting low rates of multidrug-resistant (MDR) bacteria. This single center study analyzes the results of NHAP patients admitted through the Emergency Department (ED) at a tertiary center during the year 2010. There were 116 cases, male gender corresponded to $34.5 \%$ of patients and median age was 84 years old (IQR 77-90). Comorbidities were present in $69.8 \%$ of cases and $48.3 \%$ of patients had used healthcare services during the previous 90 days. In-hospital mortality rate was $46.6 \%$ and median length-of-stay was 9 days. Severity assessment at the Emergency Department provided CURB65 index score and respective mortality (\%) results: zero: $n=0$; one: $n=7$ (0\%); two: $n=18$ (38.9\%); three: $n=26$ (38.5\%); four: $n=30(53.3 \%)$; and five; $n=22(68.2 \%)$; and sepsis $n=50$ (34.0\%), severe sepsis $n=43(48.8 \%)$ and septic shock $n=22$ $(72.7 \%)$. Significant risk factors for in-hospital mortality in multivariate analysis were polypnea $(p=0.001)$, age $\geq 75$ years $(p=0.02)$, and severe sepsis or shock $(p=0.03)$ at the ED. Microbiological testing in $78.4 \%$ of cases was positive in $15.4 \%(n=15)$ : methicillin-
\end{abstract}

Rui Pereira

rui.pereira@mail.com

1 Intensive Care Unit, Hospital Curry Cabral, CHLC, Lisbon, Portugal

2 Medicina Interna 4, Hospital Santa Marta, CHLC, Lisbon, Portugal resistant Staphylococcus aureus (26.7\%), Pseudomonas aeruginosa (20.0 \%), S. pneumoniae (13.3\%), Escherichia coli $(13.3 \%)$, others $(26.7 \%)$; the rate of MDR bacteria was $53.3 \%$. This study reveals high rates of mortality and MDR bacteria among NHAP hospital admissions supporting the use of empirical broad-spectrum antibiotic therapy in these patients.

Keywords Pneumonia - Nursing home $\cdot$ Long-term · Emergency department $\cdot$ CURB65 $\cdot$ Sepsis

\section{Introduction}

Pneumonia is one of the most common infections arising amongst nursing home residents [1]. Nursing home-acquired pneumonia (NHAP) is a concept emerging from within the definition of Health-case associated Pneumonia (HCAP) and is an entity frequently presenting to the Emergency Department (ED) requiring hospital admission. Patients suffering from this condition are typically of older age, and present a high burden of comorbid conditions. The presence of multi-resistant drug (MRD) pathogens, especially methicillin-resistant Staphylococcus aureus (MRSA) and Pseudomonas aeruginosa, is a major concern [2]. For these reasons increased mortality is expected when compared to other types of pneumonia.

The Portuguese population has importantly aged with $19.3 \%$ of the people in the age group $\geq 65$ years old, representing a $19.0 \%$ relative increase between 2001 and 2011 [3]. Furthermore the incidence of Community-acquired Pneumonia (CAP) hospital admissions has also been reported to have increased $28.2 \%$ between the first and the second half of the past decade [4]. Therefore, the number of nursing home residents and the frequency of NHAP 
should also be expected to increase, although current data on its incidence are scarce.

The authors aimed to analyze incidence, microbiological results, outcomes and risk factors in NHAP admissions at a tertiary hospital center during the year 2010 .

\section{Methods}

The was a retrospective cohort study of all adult patients admitted through the Emergency Department (ED) with a primary diagnosis of Pneumonia at Centro Hospitalar Lisboa Central (CHLC), E.P.E., a Portuguese tertiary Hospital Center located in Lisbon's metropolitan area, during the period between 1st January and 31st December of 2010.

Pneumonia diagnostic codes (International Classification of Diseases-9: 480-488) were searched for in the institutional database. "Pneumonia" was defined as hospital admission for acute lower respiratory tract infection associated with de novo radiographic shadowing during the initial $48 \mathrm{~h}$ for which there was no other explanation, and was clinically managed as such [5].

Pneumonia episodes were screened for clinical and radiological criteria and selected if these were fulfilled to build a cohort. Patients with nursing home residence were then screened and included in this study. Hospital discharge in the previous 10 days was an exclusion criteria considering re-admission of the same illness or nosocomial pneumonia etiology.

"Comorbidity" was considered as any prior history of chronic organic illness, solid or hematologic neoplasm and "anemia": hematocrit <30\%. For risk factor analysis "Confusion" was considered as any acutely altered state of conscience; "polypnea": respiratory rate $>20$ cycles/min or clinical reference to dyspnea, polypnea, or tachypnea; "hypoxemia": peripheral blood oxygen saturation $<90 \%$ or arterial blood oxygen pressure $<60 \mathrm{mmHg}$; "tachycardia": cardiac rate $>125$ beats/min; "hypotension": systolic blood pressure $<90 \mathrm{mmHg}$ or diastolic $<60 \mathrm{mmHg}$; and "nursing home": patient resident at a non-hospital longterm care facility.

Microbiological results considered cultural results from products obtained during the initial $48 \mathrm{~h}$ from ED admission, as well as urinary antigens for Legionella pneumophila and Streptococcus pneumoniae and H1N1 influenza PCR throughout the entire hospital stay.

True bacteriemia was considered as described by Weinstein et al. and cited in the literature [6].

All clinical and laboratory results were obtained from the initial approach at the ED, as well as severity of illness scoring using CURB65 index score [7] and the revised Sepsis classification criteria [8].
To compare NHAP CURB65 discriminative ability for in-hospital death prediction we used data from the group of CAP patients derived from the same original cohort [9].

Statistical tests for data analysis included binomial test for proportion, Fisher's exact Chi-square test for contingency tables, Mann-Whitney test for median values comparison, Kruskal-Wallis test for multiple independent variable and receiver operator curve (ROC) for CURB65 index score analysis. Risk associated variables with $p$ value $\leq 0.15$ were included in multivariable analysis. Binary logistic regression using backward method with probability for stepwise removal $>0.1$ was used for multivariable analysis. Area under the curve (AUC) and significance of the difference between the areas under two independent ROC Curves were analyzed as cited in the literature [10, 11]. Statistical significance was considered when double sided $p$ value was $\leq 0.05$. Confidence intervals of $95 \%$ (CI $95 \%$ ) were used for odds ratio (OR) and AUC. Statistical software OpenEpi ${ }^{\circledR}$ (Center for Disease Control and Prevention, Atlanta, GA, EUA) and "Statistical Package for the Social Sciences” v17.0. (IBM SPSS ${ }^{\circledR}$, Ill. EUA) were used for data analyzes.

The study was approved by the Institution's Ethics Committee and Administration.

\section{Results}

\section{Patient selection}

During the study period there were 29,684 adult hospital admissions from which 1635 had a Pneumonia diagnosis. Eight hundred and eighty two cases were excluded due to: alternative primary admission diagnosis $(n=272)$, lack of digital clinical records $(n=169)$, repetition $(n=140)$, hospital discharge within the 10 previous days $(n=85)$, absence of radiologic shadowing $(n=65)$, radiologic exams unavailable $(n=25)$ and other reasons $(n=125)$ including unknown reason for admission, patient not admitted via ED and age under 15 years old.

There were 753 cases of hospital admission due to pneumonia selected for this cohort and 116 cases of NHAP were included in this study analysis [9].

\section{Epidemiology}

Temporal distribution of cases showed bimodal peak incidence in March and July (both $12.1 \%$ ) and low in May and December (both $1.7 \%$ ).

Male gender corresponded to $34.5 \%(n=40)$ of the study sample $(p<0.001)$. Median age was 84 years old [interquartile range (IQR) 77-90]. Male median age was 
80 years old (IQR 75-86) while female median age was 87 (IQR 83-91).

There were comorbidities in $69.8 \%(n=81)$ of cases, including heart failure $(n=35)$, cerebral vascular disease $(n=33)$, anemia $(n=30)$, diabetes mellitus $(n=22)$, lung disease $(n=17)$, renal failure $(n=13)$, history of neoplasm $(n=9)$ and liver disease $(n=1)$.

During the prior 90 days (admissions within the prior 10 days excluded) $48.3 \%$ of these patients had reportedly had healthcare services: $34.5 \%$ had been to ED consultation, $27.6 \%$ had taken antibiotic therapy [penicillin derivates $(n=19)$, cephalosporin $(n=7)$, quinolone $(n=3)$, sulfonamides $(n=2)$ and macrolide $(n=1)]$ and $20.7 \%$ had had hospital admission stay.

Patient characteristics are described in Table 1.

\section{Microbiology}

Microbiological testing for etiology was performed in $78.4 \%(n=91)$ of cases yielding a positive result in $15.4 \%$

Blood cultures were performed in 83 cases with bacteriemia in $12.0 \%$ and contamination in $2.4 \%$. Respiratory secretions were collected for culture in 17 cases with agent isolation in $23.5 \%$, contamination in $23.5 \%$ and inadequate sampling in $23.5 \%$. Urinary antigens for $L$. pneumophila and Streptococcus pneumoniae were searched in 30 and 26 cases, respectively, and H1N1 Influenza virus

Table 1 Nursing home-acquired pneumonia patient characteristics

\begin{tabular}{lcl}
\hline$n=116$ & $\%$ & $n$ \\
\hline Female gender & 65.5 & 76 \\
Age (years, median) & 84.0 & IQR 77-90 \\
Hospital stay (days, median) & 9.0 & IQR 5-13 \\
Comorbidities & 69.8 & 81 \\
Cardiac & 30.2 & 35 \\
Cerebrovascular & 28.4 & 33 \\
Anemia & 25.9 & 30 \\
Diabetes mellitus & 19.0 & 22 \\
Pulmonary & 14.7 & 17 \\
Renal & 11.2 & 13 \\
Neoplasm & 7.8 & 9 \\
Liver & 0.9 & 1 \\
Previous healthcare contact & 48.3 & 56 \\
ED consult & 34.5 & 40 \\
Antibiotic therapy & 27.6 & 32 \\
Hospitalization & 20.7 & 24 \\
Overall mortality (\%) & 46.6 & 54 \\
\hline
\end{tabular}

$I Q R$ interquartile range, $E D$ emergency department respiratory swab was taken for PCR analysis in two cases with no positive results.

Identified etiologic agents $(n=15)$ were: MRSA (26.7\%), Pseudomonas aeruginosa (20.0\%), S. pneumoniae (13.3\%), Escherichia coli (13.3\%), Haemophilus influenzae (6.7\%), Enterococcus faecalis (6.7\%), Staphylococcus capitis $(6.7 \%)$ and Streptococcus agalactiae $(6.7 \%)$. P. aeruginosa and H. influenzae isolations were obtained from respiratory secretions.

Of these isolated agents $53.3 \%$ were MDR pathogens [four MRSA, three $P$. aeruginosa and one extended spectrum beta-lactamase (ESBL) producing E. coli].

\section{Hospital results}

Overall in-hospital mortality rate was $46.6 \%(n=54)$. Median length-of-stay was 9 days $(\mathrm{P} 25=5$; P75 $=13)$.

Male mortality was $40.0 \%$ and female $50.0 \%$. The median of age for the deceased was 86 years old (IQR 82-91) and 83 (IQR 75-88) for survivors. Mortality in the presence of comorbidities was $45.7 \%$ versus $48.6 \%$ in its absence. Comparison of survivor and non-survivor characteristics is described in Table 2.

Sepsis criteria were confirmed in 115 cases with $43.1 \%$ sepsis, $37.1 \%$ severe sepsis and $19.0 \%$ septic shock and matching mortality rates of $34.0,48.8$ and $72.7 \%$.

Severity of illness case distribution by CURB65 index score $(n=103)$ and respective 30 day mortality rates, illustrated in Graphic 1, were as follows: zero $(n=0)$; one $(n=7), 0 \%$; two $(n=18), 38.9 \%$; three $(n=26)$, $38.5 \%$; four $(n=30), 53.3 \%$; and five $(n=22) ; 68.2 \%$ ( $p=0.02$ between groups);with a respective ROC AUC of 0.673 (IC $95 \%$ 0.569-0.776).

When comparing CURB65 AUC ROC discriminative ability for outcome prediction in NHAP to the CAP group (0.789; IC $95 \% 0.724-0.854 ; n=314)$ from the same cohort the result was not significantly inferior $(p=0.1)$.

\section{Risk factors}

Significant risk factors for in-hospital death in univariate analysis were polypnea $(p<0.001)$, hypotension $(p=0.04)$, severe sepsis or shock $(p=0.01)$, age $\geq 75$ years $(p=0.04)$ (Table 2$)$. In multivariable analysis association with mortality was significant for variables polypnea $(p=0.001)$, age $\geq 75$ years $(p=0.02)$, and severe sepsis or shock $(p=0.03)$ (Table 3$)$.

The presence of MDR pathogens was associated in univariate analysis with severe sepsis or shock $(p=0.04)$, hypotension $(p=0.04)$ and confusion $(p=0.04)$. In multivariate analysis none of these associations reached significance. 
Table 2 Nursing homeacquired pneumonia survivor vs. non-survivor characteristics comparison

Graphic 1 CURB65 index score and 30 day in-hospital mortality distribution of nursing home-acquired pneumonia patients

\begin{tabular}{|c|c|c|c|c|c|}
\hline & \multicolumn{2}{|c|}{ Survivors $(n=62)$} & \multicolumn{2}{|c|}{ Non-survivors $(n=54)$} & \multirow[t]{2}{*}{$p$ value } \\
\hline & $n$ & $\%$ & $n$ & $\%$ & \\
\hline Male gender & 24 & 38.7 & 16 & 25.8 & 0.33 \\
\hline Age (years; median) & 83 & IQR 75-88 & 86 & IQR 82-91 & 0.01 \\
\hline Hospital stay (days; median) & 10 & IQR 8-15 & 5 & IQR 2-10 & $<0.001$ \\
\hline Comorbidities (overall) & 44 & 71.0 & 37 & 68.5 & 0.84 \\
\hline Cardiac & 17 & 28.3 & 18 & 34.6 & 0.54 \\
\hline Cerebrovascular & 21 & 34.4 & 12 & 23.5 & 0.22 \\
\hline Anemia & 13 & 21.0 & 17 & 32.7 & 0.20 \\
\hline Diabetes mellitus & 15 & 24.2 & 7 & 13.0 & 0.16 \\
\hline Pulmonary & 9 & 15.0 & 8 & 16.0 & 1.00 \\
\hline Renal & 5 & 8.3 & 8 & 15.1 & 0.38 \\
\hline Neoplasm & 3 & 4.9 & 6 & 11.3 & 0.30 \\
\hline Liver & 0 & & 1 & 2.0 & \\
\hline Previous healthcare contact & 30 & 48.4 & 26 & 48.1 & 1.0 \\
\hline ED consultation & 20 & 32.3 & 20 & 37.7 & 0.56 \\
\hline Antibiotic therapy & 19 & 31.1 & 13 & 26.0 & 0.67 \\
\hline Hospitalization & 11 & 17.7 & 13 & 24.1 & 0.49 \\
\hline Confusion & 37 & 59.7 & 38 & 73.1 & 0.17 \\
\hline Urea $>40 \mathrm{mg} / \mathrm{dL}$ & 44 & 72.1 & 43 & 81.1 & 0.18 \\
\hline Polypnea & 24 & 43.6 & 41 & 78.8 & $<0.001$ \\
\hline Hypotension & 20 & 32.3 & 28 & 51.9 & 0.04 \\
\hline Age $>75$ years & 48 & 77.4 & 50 & 92.6 & 0.04 \\
\hline Tachycardia & 37 & 59.7 & 39 & 72.2 & 0.17 \\
\hline Creatinine $>1.20 \mathrm{mg} / \mathrm{dL}$ & 18 & 29.0 & 23 & 44.2 & 0.12 \\
\hline Severe sepsis/shock & 28 & 45.2 & 37 & 68.5 & 0.01 \\
\hline
\end{tabular}

$I Q R$ interquartile range, $E D$ Emergency Department

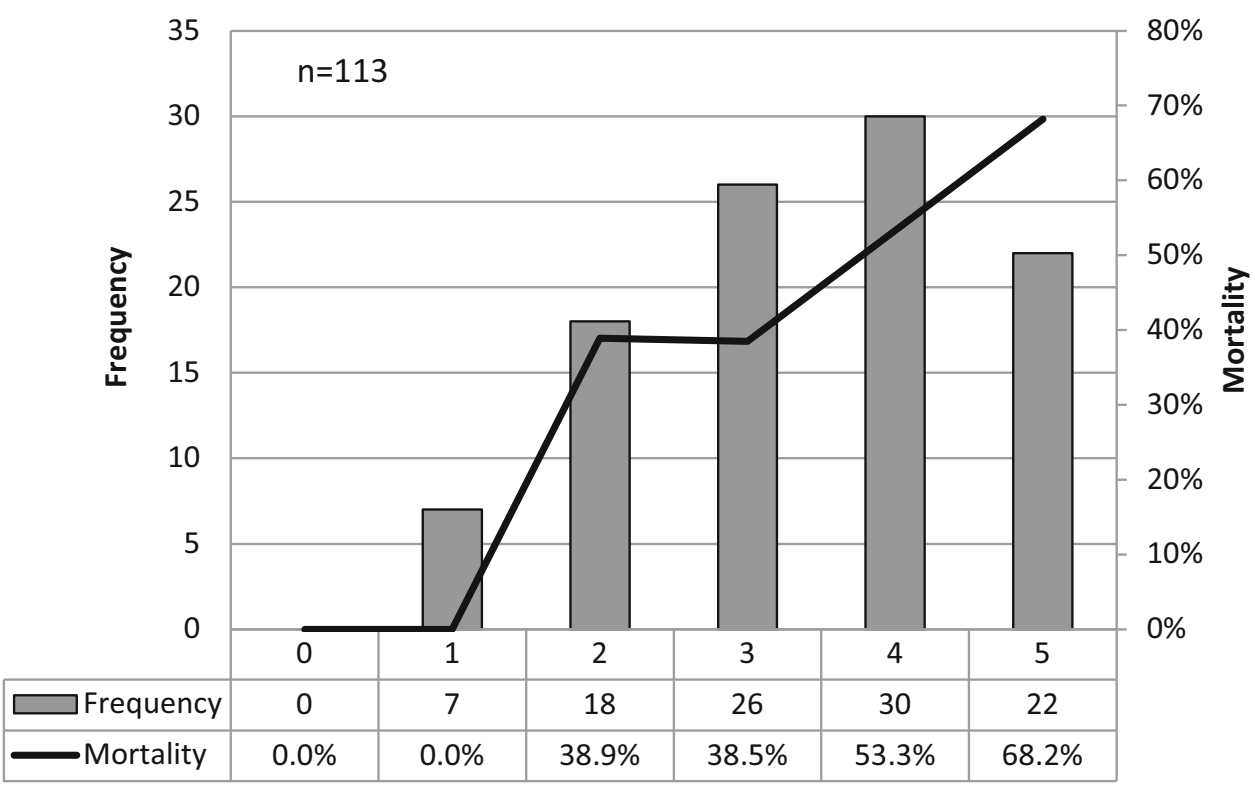


Table 3 Multivariable backward stepwise logistic regression for inhospital mortality associated risk factors

\begin{tabular}{llllr}
\hline & $p$ value & Odds ratio & \multicolumn{2}{l}{$95 \%$ CI } \\
\hline Polypnea & 0.001 & 5.1 & 2.0 & 12.7 \\
Age $\geq 75$ years & 0.02 & 4.5 & 1.2 & 16.6 \\
Severe sepsis or shock & 0.03 & 2.7 & 1.1 & 6.6 \\
Creatinine $>1.20 \mathrm{mg} / \mathrm{dL}$ & 0.08 & 2.3 & 0.9 & 5.9 \\
\hline
\end{tabular}

Two step analysis. Variable hypotension removed from model due to $p$ value 0.93 in the first step

\section{Limitations}

There are limitations to this study, namely the absence of (1) a standard clinical procedure protocol, including (2) serological or PCR research for atypical etiologic agents; (3) antibiotic therapy prescription analysis, (4) time interval analysis between ED admission and antibiotic administration (these last two with an impact on survival), (5) potential admission bias and (6) single center data precluding the generalizability of results.

\section{Discussion}

During the study period Hospital Center Lisboa Central (CHLC) included hospitals São José, Santo António dos Capuchos and Santa Marta caring for a population of approximately 191.000 adults with a high proportion (24.2\%) of people aged $\geq 65$ years old [3].

In our study the typical NHAP patient profile was rendered by a clear predominance of the female gender, advanced age and preexisting comorbidities, with more than one-third of the study population having had healthcare services during the prior 3 months indicating a somewhat expected decaying health status and high use of healthcare resources.

Hospital results showed high mortality rates particularly among the most advanced ages and most severely ill patients, which could be interpreted in the light of the vulnerability of this population due to comorbidities and extremes of age conveying limited organic functional reserve and end-of life pneumonia. In selected cases admission to Intensive Care and mechanical ventilation may have been limited although this was not assessed in our study.

Clinical severity of illness assessment tools, Sepsis criteria and CURB-65, were useful at the ED discriminating risk groups for outcome. Several studies have demonstrated the usefulness of CURB65 in NHAP and HCAP [12-14] although it may show reduced discriminating ability as compared to its original use in CAP populations. This is demonstrated in our study by a non- significant inferior CURB65 ROC AUC comparison result between NHAP and CAP groups derived from the same original cohort [9].

Multivariate analysis for significant in-hospital mortality risk factors revealed association with polypnea, age $\geq 75$ years old and severe sepsis or shock at the ED all of which is to be expected. Regarding risk factors for isolation of MDR pathogens no significant associations were unveiled possibly due to the small number of patients.

The most frequently isolated agents in this study were MRSA and P. aeruginosa and over half of all isolated bacteria were MDR. This observation is of the utmost importance due to implications on initial patient approach and initial empirical therapy.

Previous fundamental works have addressed NHAP within the HCAP definition considering high risk of MDR pathogens, namely MRSA and $P$. aeruginosa, and recommend empirical treatment with broad-spectrum antibiotic therapy, as opposed to CAP [18, 19]. A major concern regarding this approach is the overuse of empirical broadspectrum antibiotics and the development of further bacterial resistance to these therapeutical agents.

Recent studies report $S$. pneumoniae as one of the principal etiological NHAP agent, as well as indicate a low prevalence of MDR pathogens and question the recommendation to treat these patients with empirical broadspectrum antibiotics [15-18].

Research data from both HCAP and NHAP populations have provided with heterogeneous microbiological results and thus failed so far to produce changes in current treatment recommendations. The issue here concerned may be the importance of understanding local realities and adapting to them. It is possible that part of the heterogeneity of microbiological results in NHAP studies reflect differences in study methodologies and regional contrasts regarding socio-economic and healthcare factors affecting nursing home resident populations (What is the nursing home user profile? How is the quality of nursing and medical assistance and antibiotic prescription?). Our study aimed to analyze NHAP from an in-patient perspective and does not focus on the questions regarding the out-patient and the quality of nursing home care.

We believe that current NHAP recommendations for aggressive empirical broad-spectrum antibiotic therapy should apply to our study population as suggested by the high rates of mortality and MDR bacteria observed. The importance of clinical pro-active etiological investigation to guide individual treatment options and provide epidemiologic data must be stressed at this point. NHAP should be regarded as a separate entity from HCAP and investigation (including both in-patients and out-patients to avoid admission bias) should be encouraged for the development of tailored therapeutic strategies. 


\section{Conclusion}

In this NHAP study in-hospital mortality was very high and associated with extremes of age, polypnea and severe sepsis or shock at the ED. The most frequent pathogens were MRSA and $P$. aeruginosa and the majority of isolated bacteria were MDR supporting the recommendations for empirical broad-spectrum antibiotic therapy. Understanding NHAP as a separate entity is crucial and further research should lead to the development of tailored therapeutic strategies.

Acknowledgments The authors would like to thank for the contribution of Ana Catarina Patrício, Filipa Silva, Joana Ferreira, Pedro la Féria, Pedro Silva, João Oliveira, Lara Câmara, Rui Malheiro, Rodrigo Leão, Mário Silva, Pedro Russo, Ana Lladó and Paulo Barreto.

\section{Compliance with ethical standards}

Conflict of interest The authors declare that they have no conflict of interest.

Statement of human and animal rights The study was approved by the Institution's Ethics Committee and Administration. All human ethical standards were respected in the study.

Informed consent Informed consent was waved due to complete patient anonymity.

\section{References}

1. Mylotte JM (2002) Nursing home-acquired pneumonia. Clin Infect Dis 35:1205-1211

2. Cillóniz C et al (2013) Impact of age and comorbidity on cause and outcome in community-acquired pneumonia. Chest 144:999-1007

3. Carvalho A, de C. Censos (2012) Resultados definitivos. Portugal, 2011. 559. http://censos.ine.pt. http://www.ine.pt/investiga dores/Quadros/Q102.zip. Accessed 25 Feb 2016

4. Froes F, Diniz A, Mesquita M, Serrado M, Nunes B (2013) Hospital admissions of adults with community-acquired pneumonia in Portugal between 2000 and 2009. Eur Respir J 41:1141-1146

5. Lim W, Baudouin S, George R (2009) BTS guidelines for the management of community acquired pneumonia in adults: update 2009. Thorax 64:iii7
6. Weinstein MP et al (1997) The clinical significance of positive blood cultures in the 1990s: a prospective comprehensive evaluation of the microbiology, epidemiology, and outcome of bacteremia and fungemia in adults. Clin Infect Dis 24:584-602

7. Lim WS et al (2003) Defining community acquired pneumonia severity on presentation to hospital: an international derivation and validation study. Thorax 58:377-382

8. Dellinger RP et al (2013) Surviving sepsis campaign: international guidelines for management of severe sepsis and septic shock: 2012. Crit Care Med 41:580-637

9. Rui Pereira, Lladó A, Silva P, Almeida A, Patrício AC, Silva F, Ferreira J, Oliveira J, Câmara L, Leão R, Malheiro R, Oliveira S, Silva M, Russo P, Féria P, Barreto P. Internamentos hospitalares por pneumonia adquirida na comunidade num centro hospitalar. Comunicação Oral CO-09-03. 20 $0^{\circ}$ Congresso Nacional de Medicina Interna. http://www.spmi.pt/20congresso/resumos_ aceites_consulta.php?id=CO-09-03. Accessed 25 Feb 2016

10. Hanley JA, McNeil BJ (1982) The meaning and use of the area under a receiver operating characteristic (ROC) curve. Radiology 143:29-36

11. Fischer JE, Bachmann LM, Jaeschke R (2003) A readers' guide to the interpretation of diagnostic test properties: clinical example of sepsis. Intensive Care Med 29:1043-1051

12. Ugajin M, Yamaki K, Hirasawa N, Kobayashi T, Yagi T (2014) Prognostic value of severity indicators of nursing-home-acquired pneumonia versus community-acquired pneumonia in elderly patients. Clin Interv Aging 9:267-274

13. Lee J-C et al (2013) Comparison of severity predictive rules for hospitalised nursing home-acquired pneumonia in Korea: a retrospective observational study. Prim Care Respir J 22:149-154

14. Porfyridis I, Georgiadis G, Vogazianos P, Mitis G, Georgiou A (2014) C-reactive protein, procalcitonin, clinical pulmonary infection score, and pneumonia severity scores in nursing home acquired pneumonia. Respir Care 59:574-581

15. Ewig S et al (2012) Nursing-home-acquired pneumonia in Germany: an 8-year prospective multicentre study. Thorax 67:132-138

16. Brito V, Niederman MS (2009) Healthcare-associated pneumonia is a heterogeneous disease, and all patients do not need the same broad-spectrum antibiotic therapy as complex nosocomial pneumonia. Curr Opin Infect Dis 22:316-325

17. Ma HM, Wah JLS, Woo J (2012) Should nursing home-acquired pneumonia be treated as nosocomial pneumonia? J Am Med Dir Assoc 13:727-731

18. American Thoracic Society; Infectious Diseases Society of America (2005) Guidelines for the management of adults with hospital-acquired, ventilator-associated, and healthcare-associated pneumonia. Am J Respir Crit Care Med 171:388-416

19. Froes F et al (2014) Consensus document for the prevention of respiratory infections in adults. Rev Port Pneumol 20(2):111-114 